\title{
Efeito da intensidade luminosa sobre a capacidade de predação de larvas do peixe-rei marinho Odontesthes argentinensis
}

\author{
Effect of light intensity on prey consumption of marine pejerrey Odontesthes argentinensis larvae
}

\author{
Ricardo Vieira Rodrigues ${ }^{\mathrm{I}}$ Luciano Siqueira Freitas $^{\mathrm{I}}$ Luís André Sampaio $^{\mathrm{I}}$
}

\section{-NOTA-}

\section{RESUMO}

Este estudo teve como objetivo determinar o efeito da intensidade luminosa sobre a capacidade de predação de larvas do peixe-rei marinho Odontesthes argentinensis. Ovos fertilizados foram coletados na Praia do Cassino,Rio Grande, Rio Grande do Sul (RS). Ao completarem dois dias e posteriormente, 14 dias de vida, 15 grupos com 10 larvas foram transferidos para béqueres de $800 \mathrm{~mL}$. As larvas foram mantidas em jejum durante 12 horas nas intensidades luminosas de 0, 75, 150, 1500 e 3000lux (três repetições para cada tratamento). Logo em seguida foram oferecidos náuplios de Artemia sp. na densidade de oito náuplios $m L^{-1}$ durante 15 minutos. As larvas foram imediatamente anestesiadas e fixadas em formol $10 \%$. Posteriormente foi contado o número de náuplios presentes no trato digestório de cada larva. O número de presas ingeridas na primeira alimentação não diferiu significativamente $(P>0,05)$ entre as larvas mantidas sob 75, 150, 1500 e 3000lux, mas foi observada uma tendência de redução na ingestão de náuplios à medida que a intensidade luminosa aumenta. Na intensidade de zero lux, o consumo foi significativamente inferior $(P<0,05)$ em relação a 75, 150 e 1500lux, mas semelhante $(P>0,05)$ ao consumo das larvas mantidas a 3000lux, sugerindo que as intensidades luminosas elevadas e o escuro não são apropriados para larvas de peixe-rei recém-eclodidas. O aumento no consumo de Artemia pelas larvas de 14 dias foi proporcional ao aumento da intensidade luminosa até 1500lux, quando foi estabilizado. Considerando a capacidade de predação, este estudo indica a utilização da intensidade luminosa igual ou superior a 75lux para a alimentação inicial das larvas de O. argentinensis, entretanto a intensidade luminosa deve ser ao menos de 1500lux a partir do 14ㅇ dia de vida.

Palavras-chave: Odontesthes argentinensis, intensidade luminosa, larva, alimentação

\section{ABSTRACT}

This study evaluated the effect of light intensity on predation capacity of marine pejerrey Odontesthes argentinensis larvae. Fertilized eggs were collected at Cassino Beach (Rio Grande-RS). At two and 14 days after hatching, 15 groups of 10 larvae were transferred for $800 \mathrm{~mL}$ beakers and maintained without food during under light intensities of 0, 75, 150, 1,500 and 3,000lux (each treatment was replicated three times) during $12 \mathrm{~h}$. Immediately after that, Artemia sp. (8 nauplii $\mathrm{mL}^{-}$ $\left.{ }^{1}\right)$ were offered during $15 \mathrm{~min}$ and larvae were sampled, anaesthetized and fixed in formol $10 \%$ to count the number of nauplii in the digestive tract of each larvae. The number of prey consumed 2 days after hatching did not differ $(P>0.05)$ among larvae kept under 75, 150, 1,500 and 3,000lux, but there is a trend towards a smaller prey consumption as light intensity increases. Larvae kept in the dark consumed significantly less Artemia $(P<0.05)$ than those maintained at 75,150 and 1,500lux, but similar $(P>0.05)$ to those under $3,000 l u x$, suggesting that high light intensity and darkness are not appropriate for newly hatched larvae. Considering 14 days old larvae, Artemia nauplii consumption increased proportionally to the light intensity until 1,500lux, when it was stabilized. Therefore, it is suggested that $\boldsymbol{O}$. argentinensis larvae should be maintained under light intensity equal or higher than 75lux for the first feeding, and light intensity should be increased to 1,500lux 14 days after hatching.

Key words: Odontesthes argentinensis, light intensity, larvae, feeding

O peixe-rei, Odontesthes argentinensis, é encontrado em águas marinhas e estuarinas desde o sul do Brasil até a Argentina (BRIAN \& DYER, 2006),

'Departamento de Oceanografia, Laboratório de Maricultura, Fundação Universidade Federal do Rio Grande (FURG), CP 474, 96201-900, Rio Grande, RS, Brasil. E-mail: sampaio@mikrus.com.br. *Autor para correspondência. 
sendo um importante recurso pesqueiro nessas áreas. Essa espécie tem recebido atenção para o desenvolvimento da maricultura no sul do Brasil. Nos últimos anos, vários aspectos relacionados à sua criação foram avaliados, incluindo a reprodução, larvicultura e alevinagem (SAMPAIO, 2006).

Embora a duração do fotoperíodo afete o crescimento e a sobrevivência das larvas do peixe-rei marinho (FREITAS, no prelo), nada se conhece sobre o efeito da intensidade luminosa sobre essa espécie. A luz é um dos principais parâmetros físicos para o sucesso da alimentação dos peixes, pois suas larvas são predadoras visuais (BOEUF \& BAIL, 1999). A intensidade luminosa ideal para o desenvolvimento de larvas de peixe é alterada em função de sua idade (BOEUF \& BAIL, 1999), afetando sua capacidade de capturar presas (DOWNING \& LITVAK, 2001). Portanto, este trabalho averiguou o efeito da intensidade luminosa sobre a capacidade de predação das larvas de $\boldsymbol{O}$. argentinensis.

Ovos fertilizados de $\boldsymbol{O}$. argentinensis foram coletados na Praia do Cassino em Rio Grande, Rio Grande do Sul (RS) (32 $12^{\circ}$ ' S - 52 10 ' O). A incubação foi realizada em um tanque com $15 \mathrm{~L}$, onde a temperatura da água foi mantida em $23^{\circ} \mathrm{C}$, a salinidade em $30 \%$ e o fotoperíodo foi de 24 horas diárias de iluminação com 200lux. As larvas foram criadas nesses mesmos tanques, onde foram alimentadas ad libitum com náuplios de Artemia sp. até o momento do experimento.

$O$ efeito da intensidade luminosa foi avaliado na primeira alimentação aos dois dias de vida e aos 14 dias de vida. Para a realização dos testes, 15 grupos de 10 larvas foram transferidos para béqueres $(800 \mathrm{~mL})$, com parede e fundo pretos. As larvas foram mantidas em jejum durante $12 \mathrm{~h}$ em cinco intensidades luminosas: 0, 75, 150, 1500 e 3000lux, cada tratamento com três repetições. Após esse período de jejum, as larvas foram alimentadas com náuplios de Artemia sp. (Inve ${ }^{\circledR}$, Estados Unidos) na densidade de 8 náuplios $\mathrm{mL}^{-1}$ durante $15 \mathrm{~min}$. Em seguida, as larvas foram anestesiadas com benzocaína (50ppm) e fixadas em formol $10 \%$. Posteriormente, o número de náuplios do interior do trato digestório de cada larva foi contado, com a utilização de um microscópio estereoscópico.

$\mathrm{O}$ efeito da intensidade luminosa sobre a capacidade de predação das larvas foi analisado por meio da Análise de Variância (Uma Via), seguida do Teste Tukey com nível de significância de 95\%. Os resultados estão expressos em média \pm erro padrão.

O número de presas ingeridas por larvas de O. argentinensis na primeira alimentação foi afetado pela intensidade luminosa, sendo as maiores taxas de ingestão observadas nas intensidades de luz intermediárias (Figura 1). Resultados similares foram encontrados para larvas de Melanogrammus aeglefinus que reduzem a ingestão de presas na primeira alimentação em intensidades luminosas baixas $(0,0,5$ e 20lux) ou muito elevadas (5000 lux) (DOWNING \& LITVAK, 2001). Intensidades luminosas intermediárias durante a primeira alimentação de $\boldsymbol{O}$. argentinensis podem proporcionar melhor distinção entre a presa e a parede do tanque. Já as baixas intensidades luminosas podem provocar uma estimulação visual insuficiente, enquanto altas intensidades luminosas podem diminuir o contraste entre a presa e o tanque. Em ambos os casos a capacidade de detecção das presas fica limitada (DOWNING \& LITVAK, 2001).

Nas larvas com 14 dias de vida, as intensidades luminosas mais elevadas apresentaram os melhores resultados. A predação sobre os náuplios de Artemia sob 1500lux foi semelhante ao encontrado para 3000lux. Entretanto, na menor intensidade luminosa, a capacidade de predação foi significativamente reduzida (Figura 1). Para Gadus morhua, alta intensidade luminosa (2400lux) durante o período larval melhora o crescimento, a sobrevivência e a captura de presas, (PUVANENDRAN \& BROWN, 2002).

Os resultados deste trabalho mostram que a intensidade luminosa adequada para $\boldsymbol{O}$. argentinensis muda com a ontogenia. De fato, a capacidade visual das larvas de peixe é alterada com a ontogenia (BOEUF \& BAIL, 1999). Essas mudanças estão relacionadas a diferenciações que ocorrem na estrutura celular do olho das larvas ao longo do seu desenvolvimento (POWERS \& RAYMOND, 1990), as quais proporcionam melhor acuidade visual.

Para a maioria dos peixes a presença de luz é um fator preponderante para o sucesso da primeira alimentação. Larvas de peixe marinho submetidas a longos períodos de escuro apresentam elevada mortalidade, como é o caso do Pagrus auratus (FIELDERS et al., 2002), que morre de inanição em poucos dias. Porém, as larvas de $\boldsymbol{O}$. argentinensis conseguem predar no escuro, mesmo com menor intensidade. Esse efeito também foi observado por FREITAS, et al. (no prelo), os quais constataram que a sobrevivência das larvas criadas no escuro ou sob iluminação constante é semelhante. No entanto, esse consumo pode ser acidental, decorrente da ingestão de água envolvida na osmorregulação, ou ainda pela detecção das presas por moduladores sensoriais como quimioreceptores, neuromastos livres ou linha lateral (DOWNING \& LITVAK, 2001).

Considerando a capacidade de predação, este estudo indica a utilização da intensidade luminosa 


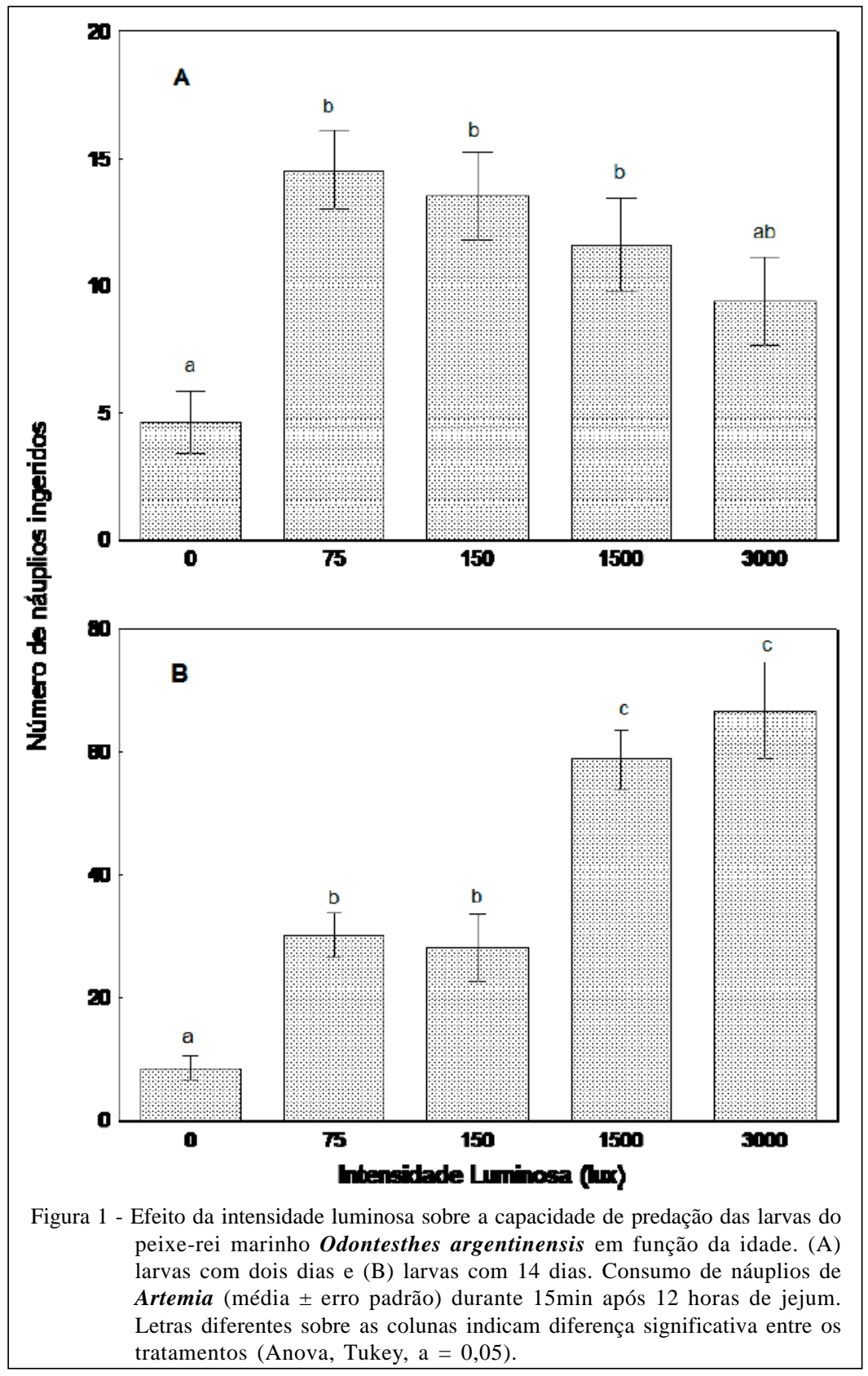

igual ou superior a 75 lux para a alimentação inicial das larvas de $\boldsymbol{O}$. argentinensis. Entretanto, a intensidade luminosa deve ser ao menos de 1500 lux a partir do $14^{\circ}$ dia de vida.

\section{AGRADECIMENTOS}

Os autores agradecem à Agência Nacional do Petróleo e Gás Natural (ANP), pela concessão da bolsa de mestrado ao estudante Ricardo V. Rodrigues, e ao Conselho Nacional de Desenvolvimento Científico e Tecnológico (CNPq), pela concessão da bolsa de produtividade em pesquisa (processo 301673/2006-3) a Luís André Sampaio.

\section{REFERÊNCIAS}

BOEUF, G.; BAIL, P.-Y.L. Does light have an influence on fish growth? Aquaculture, v.177, p.129-152, 1999.

BRIAN, S.; DYER, H. Systematic revision of the South American silversides (Teleostei, Atheriniformes). Biocell, v.30, n.1, p.69-88, 2006.

DOWNING, G.; LITVAK, M.K. The effect of light intensity and espectrum on the incidence of first feeding by larval haddock. Journal of Fish Biology, v.59, p.1566-1578, 2001.

Ciência Rural, v.39, n.1, jan-fev, 2009. 
FIELDERS, D.S. et al. Effect of photoperiod on growth and survival of snapper Pagrus auratus larvae. Aquaculture, v.211, p.135-150, 2002.

FREITAS, L.S. et al. The effects of photoperiod on survival and growth of the marine pejerrey Odontesthes argentinensis larvae. Aquaculture Research, no prelo. Disponível em: http://www3.interscience.wiley.com/cgi-bin/fulltext/ 121358615/HTMLSTART. doi: 10.1111/j.13652109.2008.02080.x

POWERS, M.K.; RAYMOND, P.A. Development of visual system. In: DOUGLAS, R.H.; DJAMGOZ, M.B.A. The visual system of fish. London: Chapman and Hall, 1990. Cap.12, p.419-422.

PUVANENDRAN, V.; BROWN, J.A. Foraging, growth and survival of Atlantic cod larvae reared in different light intensities and photoperiods. Aquaculture, v.214, p.131151, 2002.

SAMPAIO, L.A. Production of 'pejerrey' Odontesthes argentinensis fingerlings: a review of current techniques. Biocell, v.30, p.121-123, 2006 . 